\title{
Mechanics of Deepwater Steel Catenary Riser
}

\author{
Menglan Duan ${ }^{1}$, Jinghao Chen ${ }^{1}$ and Zhigang $\mathrm{Li}^{2}$ \\ ${ }^{1}$ Offshore Oil/Gas Research Center, China University of Petroleum, Beijing, \\ ${ }^{2}$ Offshore Oil Engineering Co., Ltd., Tianjian, \\ P. R. China
}

\section{Introduction}

With the exploration and development of oil and gas expanded to deepwater area, many new floating structures are developed to reduce the cost. Steel catenary Riser (SCR) is a flexible steel pipe that conducts well fluids from the subsea wellhead to the production floating vessel. SCR has the advantages of low manufacturing cost, resistance of high temperature and high pressure, good adaptability of upper floating body's motion, etc, and is widely used in the development of deepwater oil and gas fields. Because of the complicated marine environment, SCR theory research involves fluid dynamics, nonlinear mechanics, soil mechanics and other disciplines. This chapter presents the numerical calculation for soil-riser interaction, vortexinduced vibration (VIV), fatigue, the coupling of floating vessel and riser, riser installation, etc, and provides a theoretical basis of SCR design.

\subsection{Configuration of SCR}

The static configuration of SCR is shown in figure 1.1(Kavanagh et al., 2004). SCR connects floating structure with some kinds of devices, such as flexjoint, J-tube, tapered stress joint, etc. Three kinds of connecting devices are shown in figure 1.2. In order to reduce Vortexinduced vibration (VIV), the segment of SCR is equipped with VIV suppression device(Boubenider, 2008; Taggart and Tognarelli, 2008). Figure 1.3 presents two main suppression devices of helical strake and fairing.

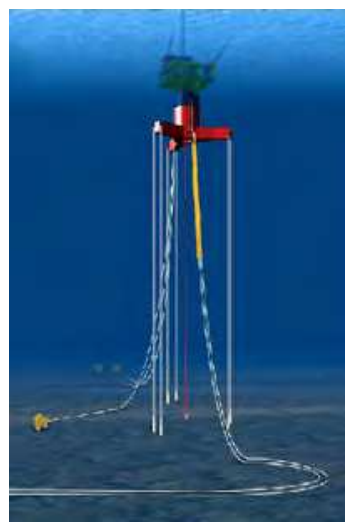

Fig. 1.1 The static configuration of SCR 


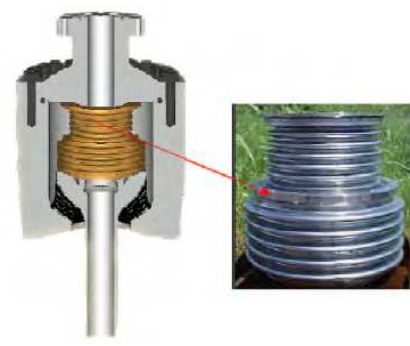

a. Flexjoint

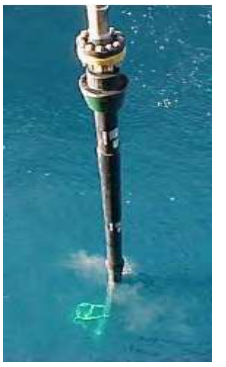

b. TSJ

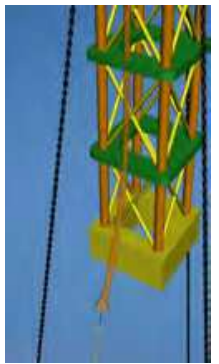

c. J-Tube

Fig. 1.2 Three kinds of connecting devices

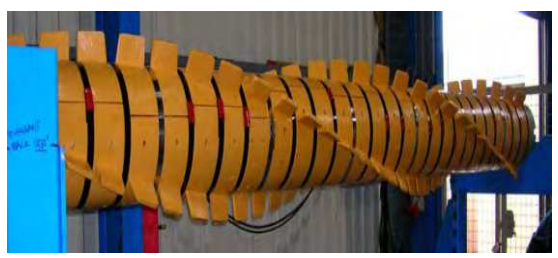

a. Helical Strake

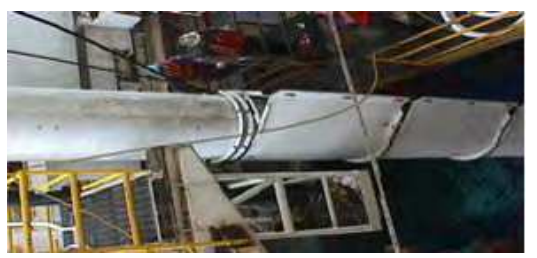

b. Fairing

Fig. 1.3 VIV suppression devices

\subsection{History of SCR development}

SCRs were initially installed on fixed platforms. Until 1994, SCRs were firstly installed on a floating platform-Auger TLP, and since then have been widely used in deepwater oil/gas fields. The main application of SCR is in Brazil, the Gulf of Mexico and West Africa on TLP, Spar and semi-submersible. Recent years, SCR is widely installed on FPSO in West Africa. This section presents some installation instances(Bai and Bai, 2005).

1994 The first SCRs were installed on Auger TLP in Gulf of Mexico.

1997 The first SCRs were installed on semi-submersible in Marlim Field.

2001 The first SCRs were installed on truss spars locate at Boomvang and Nansen fields, in Gulf of Mexico.

2004 The first SCRs were installed on FPSO vessel in West Africa.

\subsection{Analysis software for Risers}

Various riser analysis tools can be divided into two classes. One is general finite element software(Abaqus Analysis User's Manual 6.9; ANSYS ASAS Brochure), the other is professional software(Bai and Bai, 2005; Orcaflex Manual Version 9.3c).

1. General finite element software

\section{ABAQUS}

ABAQUS is developed by world famous computer simulation software company SIMULIA. It is a powerful finite element software which can solve the problem scoped from simple linear analysis to complex nonlinear analysis.

ABAQUS/Aqua is a module used in offshore industry. It includes jacket and riser analysis, J-tube pull simulations, bottom-bending calculations, and floating structure studies. 
Structures can be subjected to drag, buoyancy, and fluid inertia force under steady current and wave loadings. Wind loading is available for riser above the surface of the water.

\section{ANSYS}

ANSYS is a general purpose finite element software. It can solve structure, fluid, electric field, magnetic field, sound field and multi-physical coupling problems.

ANSYS/ASAS is a structural finite element system containing the features to meet the needs of offshore and marine engineers. ANSYS/ASAS provides the capabilities to analyze the global structures of most types of marine structures, including jackets, jack-ups, risers, offshore wind farms, and floating systems such as FPSOs, SPARs and semi-submersibles.

2. Professional software

\section{Orcaflex}

OrcaFlex is 3D non-linear time-domain finite element software developed by Orcina for static and dynamic analysis of a wide range of offshore systems, including marine risers, moorings and towed systems. It also provides some modeling elements, such as line, vessel, buoy, winch and seabed, etc. It also provides extensive graphics to assist understanding.

\section{Shear7}

Shear7 is one of the leading modeling tools for the prediction of vortex-induced vibration (VIV), developed by Professor J. Kim Vandiver in MIT. Shear7 is a mode-superposition software, which evaluates the modes likely to be excited by vortex shedding and estimates VIV response in uniform or sheared flows. It is capable of analyzing multi-mode and nonlock-in response as well as single mode lock-in response.

Other software includes Offpipe, Riflex, VIVA, Flexcom, etc.

\subsection{Overview on present research of SCR}

Mechanics of deepwater steel catenary riser is a cross-disciplinary subject that involves soil mechanics, fluid mechanics, wave mechanics and vibration mechanics. The challenge of SCR design mainly reflects three aspects: pipe-soil interaction, VIV, and coupled riser and hull.

1. Pipe-soil interaction

The touchdown zone (TDZ) is one of the key locations where the fatigue damage happens. Pipe-soil interaction affects the assessment of fatigue damage. A lot of work has been done to discover the pipe-soil interaction mechanism. Two models have been established: nondegradation model and degradation model. Non-degradation model has been applied to SCR design. Degradation model takes consideration of soil plastic deformation, water mixed, soil reconsolidation, etc. These factors affect the deformation of the trench. The establishment of degradation model is still a challenge.

2. Vortex-induced vibration

So far, the numerical simulation of VIV does not have perfect solution. The key issue is the determination of hydrodynamic force. Empirical models are obtained from experiments, which are related to empirical coefficients. The experiments at high Reynolds number should be carried out to show what new phenomena appear. Another method is CFD. Directly solving the Navier-Strokes equations requires very refined grid and micro time step. Researchers seek an approximation model. Reynolds averaged Navier-Strokes (RANS) and Large Eddy Simulation (LES) mesh more rough and save computation time, which are widely used in engineering. 
3. Coupled hull and riser

The importance of coupled analysis between riser and hull has been recognized in deepwater engineering. Considering the complexity of the coupled system, each part should be modeled mathematically. To make it more efficient, proper simulation and numerical methods should be used. Recently, the hull is taken as a 6 DOF rigid body and a slender rod theory is applied to simulating the riser and mooring line. Spring and damper are used to simulate the interaction and connection. The main difference is how they solve the coupled equation. Several engineering software have been compiled based on current research results.

The numerical simulation of SCR involved many theoretical systems and each has many branches. This chapter does not carry out a thorough study on mechanics of SCR. It only briefly introduces the numerical simulation methods commonly used in offshore industry.

\section{Pipe-soil interaction}

When the SCR is subjected to oscillating movement, there is a complex interaction between SCR and seabed. The touchdown zone (TDZ) is also the key location where SCR fatigue damage happens. Pipe-Soil interaction is the important factor that should be considered in SCR strength and fatigue analysis. Owing to the complex nonlinear behavior of soil, it's hard to establish a precise model. How to accurately simulate this interaction is still a challenge and has been a hot academic research. It affects the calculation of fatigue damage and the prediction of fatigue life.

The interaction between SCR and the seabed is affected by many factors, such as SCR properties, water entrainment, soil consolidation time, soil erosion and the development of trenching. A linear or nonlinear spring is used to model the seabed soil interacting with SCR. But linear spring does not represent the real behavior of the soil. So, recently researches on the behavior of soil interacting with SCR have focused on P-y curves of soil (where P stands for the resistance force of soil and $y$ for the vertical penetration of the SCR). Many researchers acquired the empirical equations from experiments. It can be classified into two kinds: non-degradation model and degradation model. This section presents a typical non-degradation Pipe-Soil interaction models.

\subsection{Process of pipe-soil interaction}

A typical cycle of loading-unloading-reloading is presented in Figure 2.1(Nakhaee, 2010). The P-y curve can be divided into four different paths. As shown in Figure2.2, the process of pipe-soil interaction is introduced form (a) to (e), corresponding to the stage 1-5.

Stage 1. The pipe is initially laid on virgin seabed. And there is no penetration.

Stage 2. It describes the initial penetration following along the backbone curve from Point 0 to 1 . The penetration displacement is determined by $W$ and $P . W$ is the vertical force acting on the soil (usually the submerged weight of SCR per unit length) and P is the soil resistance.

Stage 3. The pipe moves upwards and soil resistance is reduced quickly. After the soil resistance reaches zero, the loading is soil suction and increases to the peak rapidly. This process is described by P-y curve from point 1 to 2 on figure 2.1.

Stage 4. When the pipe is going on to uplift, the soil suction gradually diminishes and reaches zero from point 2 to 3 .

Stage 5. The pipe penetrates again into the soil. The resistance force follows along the curve from point 3 to 1 , which is lower than backbone curve. 


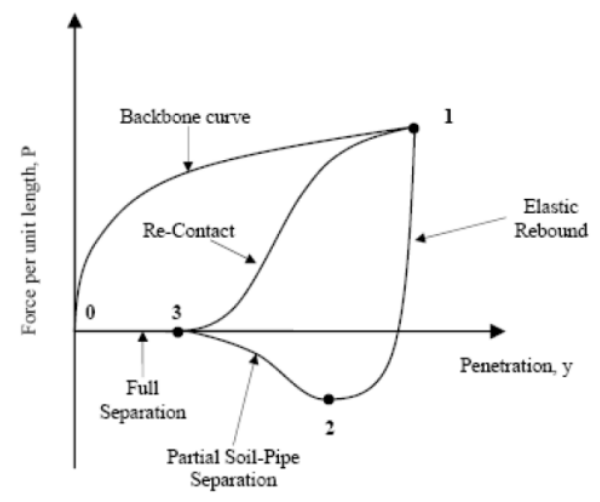

Fig. 2.1 Typical P-y curve

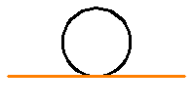

(a)

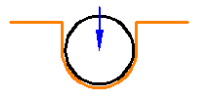

(b)

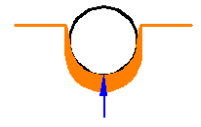

(c)

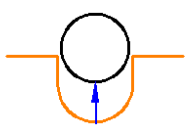

(d)

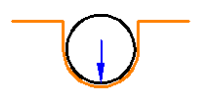

(e)

Fig. 2.2 Process of pipe-soil interaction

If the riser continues to experience the periodic loading cycle, the P-y relation will repeat the loop enclosed by the second, third and fourth path under the assumption of a nondegradation model. It should be noted that the loop area is greatly exaggerated in Figure2.1 for the purpose of demonstrating.

\subsection{Pipe-soil interaction model}

Earlier pipe-soil models are too simplified to simulate the complex interaction between pipe and soil. They assumed the seabed as rigid flat or linearly elastic spring. Many researchers investigate the mechanism of pipe-riser interaction by experiments and give various experiment equations. One famous experiment is STRIDE JIP's full scale harbor experiment(Willis and West, 2001). This experiment experiences 3 months at a harbor in the west of England. Figure 2.3 presents this test. Bridge developed advanced non-degradation models using published data and pipe-soil interaction experiments conducted by the STRIDE and CARISIMA JIP(Bridge and Laver, 2004). Bridge's models have been widely used in many Gulf of Mexico deepwater projects involving SCRs. Bridge's models are typical non-degradation model.

The process of pipe-soil interaction is introduced in 2.1. The soil force can be divided into 2 phases: soil resistance and soil suction. This part introduces the related concepts of pipe-soil interaction model.

\subsubsection{Backbone curve}

The backbone curve shows the relation between soil resistance per unit length and penetration depth for the first time. The backbone curve is typically governed by equation (2.1). 


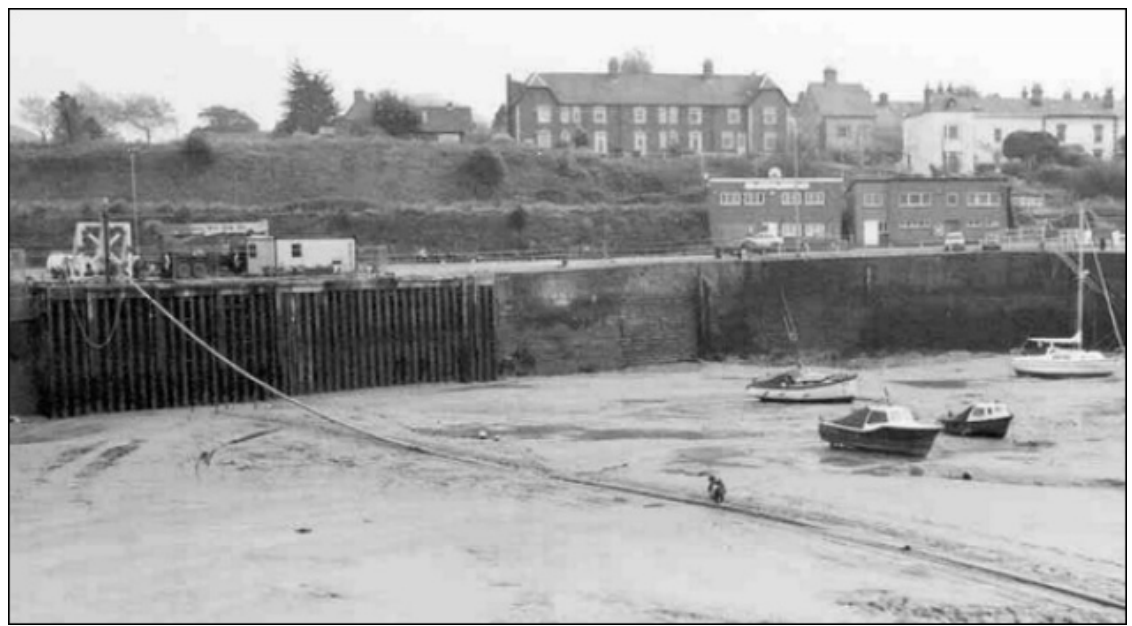

Fig. 2.3 Full scale harbor test

$$
Q_{U}=B\left(N_{C} S_{U}+\gamma Z\right)
$$

Where,

$Q_{U}$ Ultimate bearing load per unit length of pipe

$B$ Bearing width of pipe

$N_{C}$ Non-dimensional shape and depth of factor

$S_{U}$ Undrained shear strength of soil

$\gamma$ Submerged unit weight of soil

Z The depth of pipe penetration

\subsubsection{Soil resistance}

As the pipe moves vertically downward and contacts the soil, the soil presents resistance to the pipe's movement. This soil resistance is determined by soil stiffness. There are three types soil stiffness used for modeling pipe-soil interaction: static stiffness, large displacement dynamic stiffness and small displacement dynamic stiffness, as shown in figure 2.4.

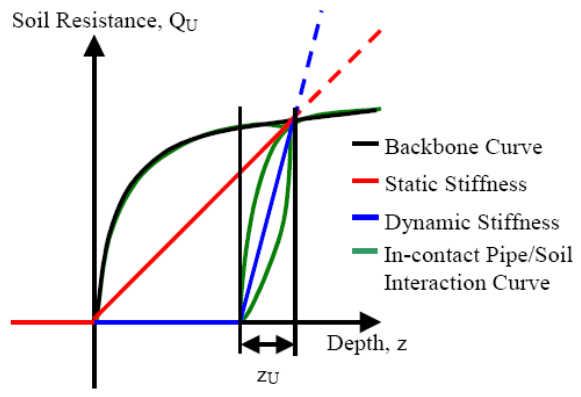

Fig. 2.4 Three soil stiffness 
1. Static stiffness

Static stiffness is used for initial penetration into the virgin seabed. The soil reaction force can be calculated using equation (2.2).

$$
R_{C}=m \sqrt{\frac{E I}{H}}
$$

Where,

$R_{C}$ Reaction force

$m$ Submerged weight of SCR per unit length

E Elastic modulus

I Moment of inertia

$H$ Tension at the TDP

2. Large displacement dynamic stiffness

Large displacement dynamic stiffness is a secant stiffness which accounts for the initial deformation of the soil. It is used to model the pipe-soil interaction where the pipe breaks out the soil. So the large displacement dynamic stiffness is calculated by equation (2.3).

$$
K=\frac{Q_{U}}{Z_{U}}=\frac{Q_{U}}{\Lambda D}
$$

Where,

$Z_{U}$ Mobilisation distance, equating $\Lambda D$

$\Lambda$ Non-dimensional parameter coming from the test

3. Small displacement dynamic stiffness

Small displacement dynamic stiffness is a hyperbolic model simulating the in-contact pipesoil interaction. So the small displacement dynamic stiffness is calculated by equation (2.4).

$$
Q=\frac{Z_{D}}{(1-X) \Lambda D+X Z_{D}} Q_{U}
$$

Where,

$Q$ Reaction force per unit length

$Z_{D}$ Dynamic displacement, the maximum value is $Z_{U}$

$X$ Soil parameter

\subsubsection{Soil suction}

When the pipe is lifted from the seabed, the soil has suction force to the pipe. Owing to the complex nonlinear behavior and multi-influence factor of soil, the suction model is mainly based on experiment. A soil suction model is based on STRIDE and CARISIMA JIP's testing as shown in figure 2.5. This soil suction model defines two parameters: maximum soil suction force $Q_{S, M A X}$ and break-out displacement $\Delta_{B}$.

Maximum soil suction force can be got from equations (2.4)-(2.6)

$$
Q_{S, M A X}=K_{C} \times K_{V} \times K_{T} \times N \times D \times S_{U}
$$




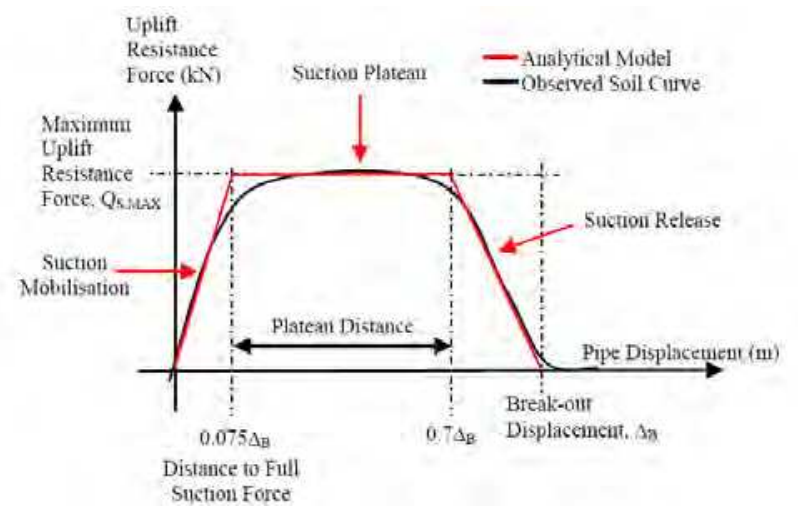

Fig. 2.5 Soil suction model

$$
\begin{gathered}
K_{V}=K_{F}\left(\frac{V}{D}\right)^{n_{F}} \\
K_{T}=K_{T F} \frac{F_{C} \sqrt{C_{V} t}}{L D^{2}}+C_{T F}
\end{gathered}
$$

Where

$K_{C}:$ Cyclic loading factor

$K_{V}:$ An empirical pull-out velocity factor

V: Pull-out velocity

$F_{C}$ : Consolidate force

$C_{V}$ : Coefficient of consolidation

$t$ : consolidation time

$K_{F}, n_{F}, K_{T F}, C_{T F}:$ Empirical constant from test data

Break-out displacement can be got from equations (2.7)-(2.9):

$$
\begin{gathered}
\Delta_{B}=K_{D V} \times K_{D T} \times D \\
K_{D V}=K_{D} \times V^{n_{D}} \\
K_{D T}=K_{D T F} \frac{F_{C} \sqrt{C_{V} t}}{L D^{2}}+C_{D T F}
\end{gathered}
$$

Where

$K_{D V}:$ An empirical break-out displacement factor

$K_{D T}:$ Consolidation time factor

$K_{D}, n_{D}, K_{D T F}, C_{D T F}$ : Empirical constant from test data

Other researchers (Aubeny and Biscontin, 2008; Nakhaee and Jun Zhang, 2007) have also done this work and established the non-degradation models. Degradation models are still ongoing in laboratory and have not yet been applied in engineering. The process of degradation models are also developed (Fontaine, et al., 2004; Nakhaee and Jun Zhang, 2009; Hodder and Byrne, 2009). 


\subsection{Conclusion}

Although many pipe-soil interaction models have been established, there is still uncertainty on the mechanism of pipe-soil interaction in TDZ. This part introduces a vertical nondegradation model developed by Bridge, which is used in offshore industry. Soil degradation, trench formation and lateral pipe-soil interaction are the problems that have never been solved. Pipe-soil interaction is the key issue for assessment of fatigue damage to which the mechanism of pipe-soil interaction is of importance.

\section{Coupling of hull and riser}

In the past, the coupling effect between the hull and mooring/riser was neglected. An uncoupled analysis which only takes the static restoring force of slender structures into account and neglects the inertial force of riser and the hydrodynamic load acting on the riser. In this way, mooring/riser is not coupled with the hull to conduct dynamic computation. But researches showed that such uncoupled analysis of TLPs, spars and FPSOs(Paulling and Webster, 1986;Zhang et al., 2008; Tahar and Kim, 2008) may be inaccurate when used in deepwater. Because as the water depth gets deeper and deeper, the inertia effect increases. So, the interaction effect greatly influences hull and line motions. The complete coupled analysis is necessary for the analysis of riser system.

The main objective of coupled analysis is to give a good estimation of floater motions. Detailed slender structure response such as the riser is secondary. The main procedure of the coupled analyses of hull and mooring/risers is as follows:

1. Establish the equation to describe the motions of hull.

2. Calculate the loads acting on the hull, including the environmental loads.

3. Establish the equation of the moor/risers. Nowadays, a discretization method is used to deal with the equation to make computation more accurate.

4. Calculate the loads acting on the mooring/risers.

5. Analyze the boundary condition and connection point of the whole system, including the connection between the platform and mooring/risers and the interaction between the risers and seabed.

6. Apply the numerical method to solve the coupled equations of hull and mooring/risers.

\subsection{Coupled hull and riser models}

A floating production system includes three parts: (1) the hull; (2) risers and mooring lines; (3) connections. To establish the coupled function of the system, every part should be modeled from mathematics.

\subsubsection{The hull model}

The hull is usually taken as a rigid body with 6 degrees of freedom. To simulate its motion, two coordinate systems are needed. One is the space fixed system $\hat{o} x \hat{y} \bar{z}$, and the other is the body- fixed system oxyz. See figure 3.1.(Chen, 2002)

According to the Newton second law, the equations of the rigid body can be written as:

$$
\begin{gathered}
m \vec{a}_{g}=\vec{F} \\
I_{g} \frac{d \vec{\omega}}{d t}+\vec{\omega} \times I_{g} \vec{\omega}=\vec{M}_{g}
\end{gathered}
$$




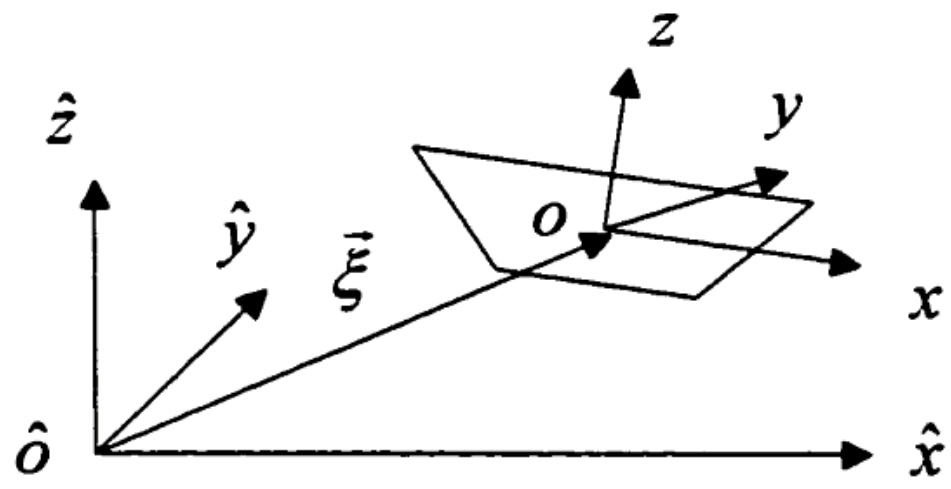

Fig. 3.12 coordinate systems of the hull

Where, $\vec{a}_{g}$ is the acceleration at the center of gravity, $I_{g}$ is the moment of inertia matrix expressed in the body-fixed coordinate system oxyz and $\vec{\omega}$ is the angular velocity also in the coordinate system oxyz. $\vec{F}$ is the resultant applied force and $M_{g}$ is the resultant applied moments.

To obtain a more specific equation, the acceleration $\vec{a}_{g}$ can be expressed in the space fixed system $\hat{o} x \hat{y} \bar{z}$ as:

$$
\vec{a}_{g}=\vec{a}_{o}+T^{t}\left(\frac{d \vec{\omega}}{d t} \times \vec{r}_{g}+\vec{\omega} \times\left(\vec{\omega} \times \vec{r}_{g}\right)\right)
$$

While the resultant moment in the body fixed system can be written as:

$$
\vec{M}=\vec{M}_{o}-\vec{r}_{g} \times T \vec{F}
$$

Inserting equation (3.3) and (3.4) into equation (3.1) and (3.2) respectively, equation (3.1) can be expressed in the space fixed system and equation (3.2) can be expressed in the body fixed system:

$$
\begin{gathered}
m \frac{d^{2} \xi}{d t^{2}}+m T^{t}\left(\frac{d \omega}{d t} \times r_{g}\right)+m T^{t}\left(\omega \times\left(\omega \times r_{g}\right)\right)=\hat{F} \\
I_{o} \frac{d \omega}{d t}+\omega \times I_{o} \omega+m r_{g} \times\left(T \frac{d^{2} \xi}{d t^{2}}\right)=M_{o}
\end{gathered}
$$

Where, superscript $t$ represents transpose of a matrix.

$\widehat{a}_{o}=\frac{d^{2} \xi}{d t^{2}}$, is the acceleration at point $o$ of the body in $\hat{o} \hat{x} \bar{y} z$

$\vec{\xi}=\left(\xi_{1}, \xi_{2}, \xi_{3}\right)^{t}$, is the displacement at point $o$ of the body in $\hat{o} x \hat{y} \bar{z}$

$\vec{\omega}=\left(\omega_{1}, \omega_{2}, \omega_{3}\right)^{l}$, is the angular velocity of the body in oxyz

$\vec{r}_{g}=\left(x_{g}, y_{g}, z_{g}\right)^{l}$, is the vector of the center of gravity (mass) of the body in oxyz 
$I_{o}$ is the moment of inertia of the body with respect to point $o$ in oxyz

$\widehat{F}$ is the total forces applied on the body in $\widehat{o} x \hat{y} z$

$\vec{M}_{o}$ is the total moments with respect to point $o$ of the oxyz coordinates

$T$ is a transfer matrix between the body-fixed coordinate system and the space-fixed coordinate system:

$$
T=\left[\begin{array}{ccc}
\cos a_{3} \cos a_{2} & \sin a_{3} \cos a_{1}+\cos a_{3} \sin a_{2} \sin a_{1} & \sin a_{3} \sin a_{1}-\cos a_{3} \sin a_{2} \cos a_{1} \\
-\sin a_{3} \cos a_{2} & \cos a_{3} \cos a_{1}-\sin a_{3} \sin a_{2} \sin a_{1} & \cos a_{3} \sin a_{1}+\sin a_{3} \sin a_{2} \cos a_{1} \\
\sin a_{2} & -\cos a_{2} \sin a_{1} & \cos a_{2} \cos a_{1}
\end{array}\right]
$$

where, $a_{1}, a_{2}, a_{3}$ are the Euler angles expressing the roll, pitch and yaw motion of the hull.

The forces acting on the hull consist of wind, wave and current loads. To be more specific, the general forces can be written as:

$$
F=F_{R}+F_{W}+F_{W D}+F_{H S}+F_{M}+F_{\text {Wind }}+F_{\text {Current }}
$$

where, $F_{R}$ represents radiation forces, $F_{W}$ exciting forces, $F_{W D}$ wave drift damping forces, $F_{H S}$ hydrostatic restoring forces, $F_{M}$ mooring/riser/tendon system forces, $F_{\text {Wind }}$ wind forces, and $F_{\text {Current }}$ is current forces.

The wave forces acting on a floating vessel are well documented in the literature. Corresponding computation methods can be referred to related thesis. For the wind and current forces, the force coefficients are usually needed which come from experiments. If the wind has mean and slowly varying components, a suitable spectrum is in need. Data from the OCIMF is useful for the FPSO analysis.

\subsubsection{Riser model}

The riser can be taken as a flexible system. It can make large displacement and angle movement with the hull. As a result, the geometric nonlinearity is a big problem for the risers. Here are some calculation methods which are used to solve the problem.

1. Linear spring method. The riser is taken as a linear spring. This method is the main method used to make frequency domain analysis. Because this method neglects too many details of the riser, the result becomes less and less accurate with the water depth increases.

2. Catenary method. This method uses the catenary equation to transform the 3-dimention to 2-dimention and fulfill the static conditions. As in method (1), the dynamic details of risers and bending stiffness are neglected.

3. Slender rod theory. Risers can be modeled by using the elastic slender rod theory. It can analyze the condition of the riser after deformation in the original coordinate system. The position of every element is expressed by a vector. Higher-order function is used to simulate the deformation of rod. But because the effect of torsion is neglected, nonvector problem of the large rotation angle deformation is not taken into account.

Method (3) is the most widely used in the offshore industry, and is illustrated in detail as follows.(Garrett, 1982; Garrett 2005)

The equation of slender rod can be written as:

$$
-\left(B r^{\prime \prime}\right)^{\prime \prime}+\left(\lambda r^{\prime}\right)^{\prime}+q=\rho \ddot{r}
$$

If the stretch of rod is assumed to be linear and small, the inextensibility condition can be approximated as: 


$$
\frac{1}{2}\left(r^{\prime} \cdot r^{\prime}-1\right)=\frac{T-T_{0}}{A E}
$$

Where, $\mathrm{B}$ is the bending stiffness (EI)

$r$ : the vector represents the centerline of the rod

$\lambda=T-B r^{\prime \prime} r^{\prime \prime}$ : Lagrange multiplier

$q:$ the applied load(weight, drag, etc)

$T$ : the tension

$T_{0}$ : the unstretched tension

$A E$ : the axial stiffness

The external forces applied on the riser include gravity, hydrostatic and hydrodynamic forces.

The gravity can be expressed as a distributed load as:

$$
q_{t}(s, t)=-\rho_{t} g A_{t} e_{y}
$$

Where, $\rho_{t}$ is riser density, $A_{t}$ is section area.

The hydrodynamic forces consist of added-mass force, drag force and Froude-Krylov force. The first two forces can be predicted by Morison's equation that will be discussed in section 4. Froude-Krylov force due to sea water outside the riser is:

$$
\vec{q}_{f}^{F-k}(s, t)=\rho_{f}\left(g \vec{e}_{y}+\overrightarrow{\ddot{u}}\right) A_{f}+\left(P_{f} A_{f} \vec{r}^{\prime}\right)^{\prime}
$$

Where,

$P_{f}$ is the pressure of sea water

$A_{f}$ is the outer cross-section area of the riser

$\rho_{f}$ is the mass density of the sea water

Due to the internal fluid, Froude-Krylov force for the riser is:

$$
\vec{q}_{i}^{F-K}(s, t)=-\rho_{i} g A_{i} \vec{e}_{y}-\left(P_{i} A_{i} \vec{r}^{\prime}\right)^{\prime}
$$

Where, $P_{i}$ is the pressure from the internal fluid; $\rho_{i}$ is the mass density of the internal fluid. $A_{i}$ is the inner area of riser.

\subsubsection{The connection}

The connection between the riser and platform can be established by using spring and damper. A linear spring can define the translational motion between platform and the top of riser. A rotational spring can define the relation between the rotation of platform and the tangential direction of the riser. For the spring, one end is at the rod element, the other one is on the rigid body. These assumptions of the connections can easily couple the motion of platform and risers numerically and simulate different kinds of connections by using different stiffness of the spring.

With the assumption of small-angle rotation, the connector forces $\left(Q_{i}\right)$ and moments $\left(L_{i}\right)$ of the spring on the end node are written as:(Arcandra, 2001)

$$
Q_{i}=K_{i}^{L}\left(X_{i}+p_{i}+\theta_{j} \times C_{j i}-r_{i}\right)
$$




$$
L_{i}=K^{\theta}\left(e_{i}+\theta_{j} D_{j i}-\frac{r_{i}^{\prime}}{\left(r_{m}^{\prime}{ }_{m}{ }^{\prime}{ }_{m}\right)^{0.5}}-\frac{r_{i}{ }^{\prime} r_{j}{ }^{\prime}}{\left(r_{n}{ }^{\prime} r_{n}{ }^{\prime}\right)^{3 / 2}}\right)
$$

Where, $K_{i}^{L}$ and $K^{\theta}$ are the stiffness matrix of linear and rotation spring

$X_{i}$ is the translational motion of the rigid body

$\theta_{i}$ is the rotational motion of the rigid body

$p_{i}$ is position vector of the point on the platform where the springs are attached

$r_{i}$ is the position vector of the node of the mooring/riser which is attached by the spring

$C_{j i}$ and $D_{j i}$ are defined to make it easier to do the numerical calculation with the position vector $p_{i}$ and the unit vector $e_{i}$ as:

$$
\begin{aligned}
& {[C]=\left[\begin{array}{ccc}
0 & -p_{3} & p_{2} \\
-p_{3} & 0 & -p_{1} \\
p_{2} & -p_{1} & 0
\end{array}\right]} \\
& {[D]=\left[\begin{array}{ccc}
0 & -e_{3} & e_{2} \\
-e_{3} & 0 & -e_{1} \\
e_{2} & -e_{1} & 0
\end{array}\right]}
\end{aligned}
$$

The resultant force $F_{i}^{S}$ and moment $M_{i}^{S}$ transferred to the body are defined as follows:

$$
\begin{gathered}
F_{i}^{S}=-Q_{i}^{S} \\
M_{i}^{S}=M_{i}^{L}+M_{i}^{\theta} \\
=Q_{k}^{S} C_{k i}+L_{k}^{S} D_{k i}
\end{gathered}
$$

Where, $M_{i}^{L}=Q_{k}^{S} \times p_{j}$ is the moment resulting from the linear spring, and $M_{i}^{\theta}=L_{k}^{S} \times e_{j}$ is the moment resulting from the rotational spring. The force $F_{i}^{S}$ and the moment $M_{i}^{S}$ act on the body.

The damper is used to control the excessive resonance of the high frequency vibration of the tensioned line like the tether or the riser in the TLP. The damper is simulated by using linear damping force proportional to the vibratory velocity of the line on the top connection node of the hull and the mooring/riser. The damping force $N_{i}^{D}$ on the connection node of the line is given by (Ran, 2000):

$$
N_{i}^{D}=C_{d}\left(\dot{X}_{i}+\dot{\theta}_{j} C_{j i}-\dot{r}_{i}\right)
$$

Where $C_{d}$ is the damping coefficient, $\dot{X}$ and $\dot{\theta}$ are the translational and rotational velocity of the rigid body, $\dot{r}$ is the velocity of the attached node of the line to the body.

The force acts on the rigid body as reaction force by:

$$
F_{i}^{D}=-N_{i}^{D}
$$

\subsection{Integrated hull and riser equations}

Compared with time domain analysis, frequency domain analysis can demonstrate directly the wave response of platform to a certain degree. So it is widely used in the theory 
computation. But in the frequency domain analysis, all nonlinearities must be linearized which may require a perturbation assumption. The time domain analysis can confirm influence of current and previous course on the mechanical state of riser at every time step. As a result to deal with all the nonlinearities in the coupled analysis, time domain is obviously the most effective way in the practical project.

The assembled equation of the coupled system of the rigid body and mooring/risers can be expressed as (HARP manuals):

$$
\left[\begin{array}{l}
{\left[\mathrm{K}^{\mathrm{L}}\right]\left[\mathrm{K}^{\mathrm{C}}\right]} \\
\hdashline\left[\left(\mathrm{K}^{\mathrm{C}}\right)^{T}\right]\left[\mathrm{K}^{\mathrm{B}}\right]
\end{array}\right]\left\{\begin{array}{l}
\mathrm{U}^{\mathrm{L}} \\
--- \\
\mathrm{U}^{\mathrm{B}}
\end{array}\right\}=\left\{\begin{array}{l}
\mathrm{F}^{\mathrm{L}} \\
--- \\
\mathrm{F}^{\mathrm{B}}
\end{array}\right\}
$$

where $\left[\mathrm{K}^{\mathrm{L}}\right]$ is the stiffness matrix of the mooring/riser and the connector springs which has $n_{L} \times\left[8 \times\left(n_{E}+1\right)-1\right]$ rows where $n_{L}$ is the total number of lines, $\left[\mathrm{K}^{\mathrm{B}}\right]$ is the stiffness matrix of the rigid body, $\left[\mathrm{K}^{\mathrm{C}}\right]$ and $\left[\left(\mathrm{K}^{\mathrm{C}}\right)^{T}\right]$ are the coupled stiffness matrices and its transpose matrix including the coupling terms of the rigid body and the mooring/riser. $\left[\mathrm{U}^{\mathrm{L}}\right]$ and $\left[\mathrm{U}^{\mathrm{B}}\right]$ are the displacement matrices of the lines and the body, $\left[\mathrm{F}^{\mathrm{L}}\right]$ and $\left[\mathrm{F}^{\mathrm{B}}\right]$ are the force and moment terms acting on the lines and the body. The size of $\left[\mathrm{K}^{\mathrm{B}}\right]$ is $6 \times 6$. $\left[\mathrm{K}^{\mathrm{C}}\right]$ has the size of $\left[8 \times\left(n_{E}+1\right)-1\right]$ rows and 6 columns per line. $n_{E}$ is the number of elements per line.

To calculate the terms of stiffness matrix, further analysis is required. And the time domain analysis consists of static analysis and dynamic analysis.

\subsubsection{Static analysis}

Before a dynamic analysis is conducted, the static problem should be solved first. Considering the geometric nonlinearity of the riser and moorings, the static analysis should be solved iteratively (Low and Langly, 2006). Here the Newton-Raphson method is applied. The hull, mooring/riser and connection are combined to form the stiffness matrix in the equation (3.19).

At each iteration step, the coupled assembly system equations are solved to obtain the behaviors for the body and lines simultaneously, and the iteration continues until the norms of the solutions reach a specified tolerance.

\subsubsection{Time domain analysis}

The time domain analysis requires integration. Several implicit methods have been developed to do it, for example, Newmark-B method, Runge-Kuta method and the AdamsMoulton method. Because the last one can solve the coupled equations of the hull and mooring/riser at every time step, it is used to integrate the nonlinear force. In this way, the terms of the stiffness matrix can be obtained from the integration. 


\subsection{Conclusion}

The coupled effect between hull and risers is usually included in the whole coupled analysis of floating production system. The whole system mainly has two components: the hull and mooring/risers. Meanwhile, the connection between platform and mooring/riser and the interaction between riser and seabed also play a part in the coupled analysis. At present, the hull is described as a 6 DOF rigid body. Risers are modeled by using a finite element representation of an elastic rod. The connection can use a combination of springs and dampers. At last, a static analysis and dynamic analysis are conducted.

\section{Fatigue}

The assessment of fatigue damage is important in SCR design. Cyclic loading can cause the fatigue damage of SCR, including wave, vortex induced vibration (VIV), hull motion, etc. This Section mainly discusses the fatigue induced by wave and VIV, and introduces fatigue assessment methods.

\subsection{Wave loading}

The method of calculating wave load on cylinder is chosen according to the size of cylinder and wavelength. SCR is a slender structure, and it mainly uses Morison's equation which has been presented by Morison etc (Morison, et al., 1950; Burrows et al., 1997). The basic assumption of this method is that the diameter of cylinder D compared with wavelength $\mathrm{L}$ is small, $\mathrm{D} / \mathrm{L}<0.2$. The cylinder cannot affect the wave field.

One cylinder stands vertically on the seabed, and the depth of water is d. Wave height is $\mathrm{H}$ and spreads along the coordinate $\mathrm{x}$. The intersection point coordinate of cylinder axis and seabed is $(\mathrm{x}, \mathrm{z})$, as shown in figure 4.1. Morison etc assumed that the horizontal wave force $f_{H}$ acting on cylinder at any height $\mathrm{z}$ consists of two parts: Water particle horizontal velocity $u_{x}$ induced force-drag force $f_{D}$; Water particle horizontal acceleration $\dot{u}_{x}$ induced force-inertia force $f_{I}$.

Morison's equation for wave loading on unit height of cylinder at any height $\mathrm{z}$ :

$$
\begin{aligned}
f_{H} & =f_{D}+f_{I} \\
& =\frac{1}{2} C_{D} \rho A u_{x}\left|u_{x}\right|+\rho V_{0} \frac{d u_{x}}{d t}+C_{m} \rho V_{0} \frac{d u_{x}}{d t} \\
& =\frac{1}{2} C_{D} \rho A u_{x}\left|u_{x}\right|+C_{M} \rho V_{0} \frac{d u_{x}}{d t}
\end{aligned}
$$

Where, $u_{x}$ is the water particle velocity at the axis center of cylinder $\frac{d u_{x}}{d t}$ is the water particle acceleration at the axis center of cylinder

$\mathrm{A}$ is the projected area of unit height of cylinder normal to the direction of wave:

$V_{0}$ is the tonnage of unit height of cylinder

$\rho$ is seawater density

$C_{m}$ is an added mass coefficient

$C_{M}$ is mass coefficient

$C_{D}$ is drag force coefficient

For a cylinder, equation (4.1) can be written as following: 


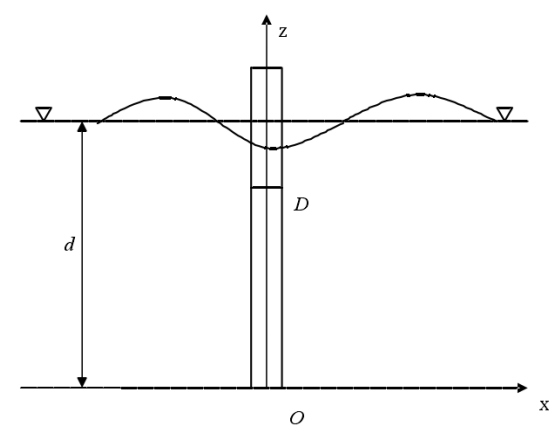

Fig. 4.1 Small scale straight cylinder coordinate system

$$
f_{H}=\frac{1}{2} C_{D} \rho D u_{x}\left|u_{x}\right|+C_{M} \rho \frac{\pi D^{2}}{4} \frac{d u_{x}}{d t}
$$

Equation (4.2) is applied for the fixed cylinder. If the cylinder moves under wave loading, the equation (4.2) should be written as following.

$$
f_{H}=\frac{1}{2} C_{D} \rho D\left(u_{x}-\dot{x}\right)\left|u_{x}-\dot{x}\right|+C_{M} \rho \frac{\pi D^{2}}{4} \frac{\partial u_{x}}{\partial t}-C_{m} \rho \frac{\pi D^{2}}{4} \ddot{x}
$$

Where, $x$ is the horizontal displacement at $z$

$\dot{x}$ is the horizontal velocity at $z$

$\ddot{x}$ is the horizontal acceleration at $z$

Equation (4.2) and equation (4.3) are used for calculating the wave force of straight cylinder. But SCR is slant cylinder, and equation (4.2) must be modified. Assuming two-dimensional wave spreads along the direction $\mathrm{x}$. At any point of cylinder, $U_{n}$ is orthogonal component of water particle velocity and $U_{t}$ is the tangent component of water particle velocity. $\dot{U}_{n}$ and $\dot{U}_{t}$ are corresponding acceleration. As a two-dimensional problem, the direction of velocity and acceleration are general not in the same line. So the Morison's equation must be written as vector. Equation (4.4) is Morison's equation for slant cylinder.

$$
f=\frac{1}{2} C_{D} \rho D U_{n}\left|U_{n}\right|+C_{M} \rho \frac{\pi D^{2}}{4} \dot{U}_{n}
$$

Where, $f$ is the vector of wave force at $z$.

The projection of $U_{n}$ to three coordinate is $\left\{U_{x}, U_{y}, U_{z}\right\}$. Assuming e is the unit vector along the axial of cylinder.

$$
e=e_{x} i+e_{y} j+e_{z} k
$$

The parameters used in Morison's equation are as following:

$$
\left\{\begin{array}{l}
U_{x}=u_{x}-e_{x}\left(e_{x} u_{x}+e_{z} u_{z}\right) \\
U_{y}=-e_{y}\left(e_{x} u_{x}+e_{z} u_{z}\right) \\
U_{z}=u_{z}-e_{z}\left(e_{x} u_{x}+e_{z} u_{z}\right)
\end{array}\right.
$$




$$
\begin{aligned}
& \left\{\begin{array}{l}
\dot{U}_{x}=\left(1-e_{x}^{2}\right) \frac{\partial u_{x}}{\partial t}-e_{z} e_{x} \frac{\partial u_{z}}{\partial t} \\
\dot{U}_{y}=-e_{x} e_{y} \frac{\partial u_{x}}{\partial t}-e_{z} e_{y} \frac{\partial u_{z}}{\partial t} \\
\dot{U}_{z}=-e_{x} e_{z} \frac{\partial u_{x}}{\partial t}+\left(1-e_{z}^{2}\right) \frac{\partial u_{z}}{\partial t}
\end{array}\right. \\
& \left|U_{n}\right|=\left(u_{x}^{2}+u_{z}^{2}-\left(e_{x} u_{x}+e_{z} u_{z}\right)^{2}\right)^{1 / 2}
\end{aligned}
$$

If slant cylinder is moving under wave load, it similar to equation (4.3).

Drag force coefficient $C_{D}$ and mass coefficient $C_{M}$ are empirical coefficients. They are from the tests, and the value of them are also depends on wave theory. The value of $\mathrm{C}_{\mathrm{D}}$ and $C_{M}$ can refer relevant standards or criterion of Classification Societies. Morison equation is the main method for calculating the wave load on small size cylinder.

\subsection{VIV}

If a cylindrical structure is subjected to the current, alternately vortex shedding will be taking place at each side of the cylinder. Vortex shedding will produce periodic force which makes cylinder vibration. When the frequency of vortex shedding is close to the nature frequency of cylinder, cylinder generates resonate. Despite decades of intensive numerical simulation and experiment research, there is not a model that can accurately simulate VIV (Williamson and Govardhan, 2008). The problem of VIV is how to determine the hydrodynamic force. Hydrodynamic force is from experiment or computational fluid dynamics (CFD), and VIV response prediction model can be classified into two categories: empirical model and CFD model.

\subsubsection{Basic concept}

VIV is affected by some parameters, such as Reynolds number, Strouhal number etc (Pan et al., 2005; Klamo, 2007). Detailed explanations of these parameters are as follows.

1. Reynolds number

The Reynolds number is a ratio of inertial force to viscous force. Its value affects the type of flow as the fluid goes through the bluff body, as depicted in figure 4.2.

$$
\operatorname{Re}=\frac{U D}{v}
$$

Where,

$U$ Fluid velocity

$v$ Coefficient of kinematic viscosity

$D$ The outer diameter of cylinder

2. Strouhal number

Strouhal number depends on the Reynolds number, which comes from experiments. Equation (4.10) is used to calculate the vortex shedding frequency.

$$
S t=\frac{f_{v} D}{U}
$$




\begin{tabular}{|c|c|c|}
\hline & $R_{e}<5$ & No separation phenomenon \\
\hline & $(5 \sim 15) \leq R_{e}<40$ & $\begin{array}{l}\text { A pair of fixed small eddy } \\
\text { behind cylinder }\end{array}$ \\
\hline & $40 \leq R_{e}<150$ & $\begin{array}{l}\text { Periodic vortex shedding in } \\
\text { laminar flow }\end{array}$ \\
\hline & $300 \leq R_{e}<3 \times 10^{5}$ & $\begin{array}{l}\text { Periodic vortex shedding in } \\
\text { turbulent flow, which can be } \\
\text { extended to 50D (cylinder } \\
\text { outer diameter) }\end{array}$ \\
\hline & $3 \times 10^{5} \leq R_{e}<3.5 \times 10^{6}$ & $\begin{array}{l}\text { Transition section. The } \\
\text { separation point is backward } \\
\text { and vortex shedding is } \\
\text { aperiodic }\end{array}$ \\
\hline & $3.5 \times 10^{6} \leq R_{e}$ & $\begin{array}{l}\text { Recovering periodic vortex } \\
\text { shedding in turbulent flow }\end{array}$ \\
\hline
\end{tabular}

Fig. 4.2 The relation between Re and vortex shedding

Where

$f_{v}$ Vortex shedding frequency

3. Reduced velocity

Reduced velocity is convenient for measuring the flow velocity. Reduced velocity is defined as:

$$
U_{r}=\frac{U}{f_{n} D}
$$

$f_{n}$ The nature frequency of cylinder

Other parameters, such as mass ratio, damping factor and aspect ratio etc are not introduced here.

\subsubsection{Empirical model}

VIV experiments involve forced or vibration of a cylinder. The empirical models are different varying with experiments. It can be divided into time domain models and frequency domain models.

\section{Time domain model}

A well known time domain model is wake oscillators which satisfy van der Pol or Rayleigh equation. Wake oscillator model couples with structure vibration and fluid wake oscillate. 


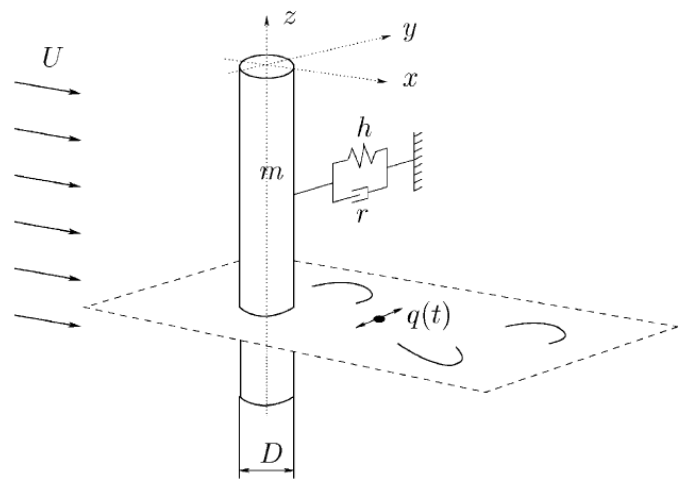

Fig. 4.3 Wake oscillator model

The 2D wake oscillator model is assumed to be an elastically supported cylinder, as shown in figure 4.3. The coupled structure and wake oscillator are described by (Facchinetti et al., 2003; Xu et al., 2010):

$$
\begin{aligned}
& \ddot{y}+\left(2 \xi \delta+\frac{\gamma}{\mu}\right) \dot{y}+\delta^{2} y=s \\
& \ddot{q}+\varepsilon\left(q^{2}-1\right) \dot{q}+q=f
\end{aligned}
$$

Where, $\delta=\frac{\Omega_{s}}{2 \pi S t(U / D)}, s=S \frac{D}{4 \pi^{2} S t^{2} U^{2} m}, f=F \frac{D}{4 \pi^{2} S t^{2} U^{2}}$,

The parameters of wake oscillator equations are explained as follows:

$y: y=Y / D: Y$ is cross-flow displacement, $D$ is outer diameter.

$q$ : Dimensionless wake variable.

$S t$ : Strouhal number.

$U$ : Fluid velocity.

$m$ : Mass accounting the mass of structure and fluid-added mass.

$\xi$ : Structure reduced damping.

$\Omega_{S}$ : Structural angular frequency.

$\gamma:$ Stall parameter.

\section{Frequency domain model}

A famous VIV analysis software Shear7 is frequency domain tool, which is based on modesuperposition method (Vandiver and Li, 2005). Its theoretical background is briefly described in the section. The governing equation is given by:

$$
m_{t} \ddot{y}+R \dot{y}-T y^{\prime \prime}=P(x, t)
$$

Where, $m_{t}$ is mass per unit length, $\ddot{y}$ is the acceleration of the structure, $R$ is the damping per unit length. $\dot{y}$ is the velocity of the structure, $T$ is the tension, $y^{\prime \prime}$ is the second derivative of the displacement of the structure with respect to the spatial variable. $P(x, t)$ is the excitation force per unit length. 
The structure displacement response can be written as the modal superposition.

$$
y(x, t)=\sum_{r} Y_{r}(x) q_{r}(t)
$$

Where, $Y_{r}(x)$ is the rth mode shape of the structure. Substituting equation (4.14) into equation (4.13) and performing modal analysis:

$$
M_{r} \ddot{q}_{r}+R_{r} \dot{q}_{r}(t)+K_{r} q_{r}(t)=P_{r}(t)
$$

Where, $M_{r}$ is modal mass, $R_{r}$ is modal damping, $K_{r}$ is modal stiffness, $P_{r}(t)$ is modal force. For pined-pined beam with varying tension, the nth natural frequency is defined by:

$$
\int_{0}^{L} \sqrt{-\frac{1}{2} \frac{T(s)}{E I(s)}+\frac{1}{2} \sqrt{\left(\frac{T(s)}{E I(s)}\right)^{2}+4 \frac{m_{t}(s) \omega_{n}^{2}}{E I(s)}}} d s=n \pi
$$

Where, $T(s)$ is tension, $E I(s)$ is the bending stiffness, $m_{t}(s)$ is the mass per unit lengh, $\omega_{n}^{2}$ is the nth natural frequency of the structure.

nth mode shape is written as:

$$
Y_{n}(x)=\sin \left(\int_{0}^{L} \sqrt{-\frac{1}{2} \frac{T(s)}{E I(s)}+\frac{1}{2} \sqrt{\left(\frac{T(s)}{E I(s)}\right)^{2}+4 \frac{m_{t}(s) \omega_{n}^{2}}{E I(s)}}} d s\right)
$$

\subsubsection{CFD model}

The CFD method obtains hydrodynamic force by solving Navier-Stokes equations directly. Laminar flow can be solved by CFD model that is identified by testing. The key issue is the establishment of turbulence model. There are 3 primary methods including Reynolds averaged Navier-Strokes (RANS), Large Eddy Simulation (LES) and direct numerical simulation (DNS). The framework of turbulent flow simulation methods is presented in figure 4.4. This section only introduces the RANS model and LES model commonly used in offshore industry.

1. RANS model

In order to solve the details of turbulent fluctuations, time-average method is usually applied. So RANS is coming up. RANS does not solve directly instantaneous Navier--Stokes equations. It decomposes flow velocity into 2 components: mean flow and fluctuating component. Time-average Navier-Strokes equations can be written as equation (4.18) (Pan et al., 2007). It describes incompressible fluid. Comparing to DNS and LES, RANS is less timeconsuming and widely used in commercial work.

$$
\left\{\begin{array}{l}
\frac{\partial u_{i}}{\partial x_{i}}=0 \\
\frac{\partial}{\partial t}\left(\rho u_{i}\right)+\frac{\partial}{\partial x_{j}}\left(\rho u_{i} u_{j}\right)=-\frac{\partial p}{\partial x_{i}}+\frac{\partial}{\partial x_{j}}\left(\mu S_{i j}-\rho \overline{u_{i}^{\prime} u_{j}^{\prime}}\right)
\end{array}\right.
$$

Where,

$u, p$ : Time-average velocity and pressure 


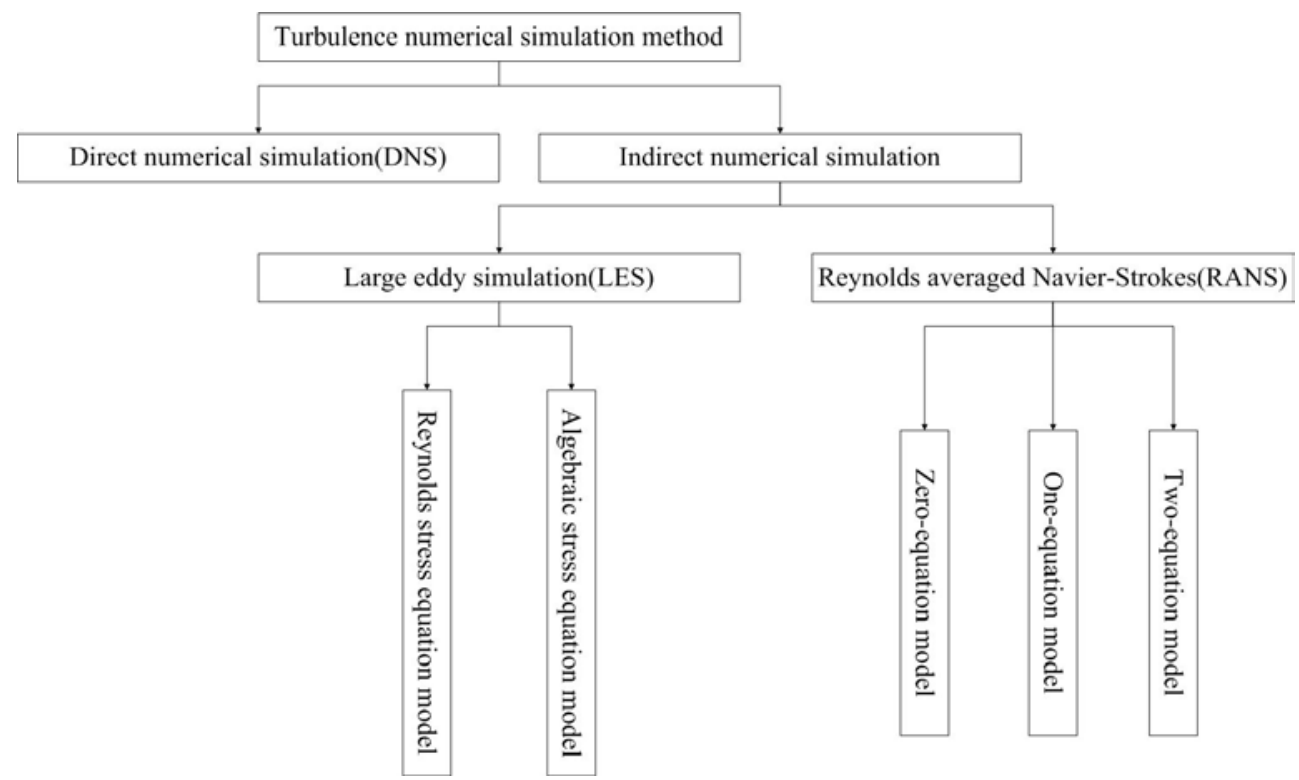

Fig. 4.4 turbulent models

$\mu$ : Molecular viscosity

$S_{i j}$ : Mean stress tensor

$\rho:$ Fluid density

The time-average equation (4.18) has addition item: $-\rho \overline{u_{i}^{\prime} u_{j}^{\prime}}$, which is called Reynolds stresses, namely:

$$
\tau_{i j}=-\rho \overline{u_{i}^{\prime} u_{j}^{\prime}}
$$

Reynolds stresses is a new unknown item. According to different assumptions, RANS can be mainly divided into 2 types: Reynolds stresses model and eddy viscosity model, as shown in figure 4.4. Recently two-equation model is extensively used in engineering. The basic two-equation model is standard $k-\varepsilon$ model, which brings in $k$ (turbulence kinetic energy) and $\varepsilon$ (turbulent dissipation rate) (Dixon and charlesworth, 2006).

Eddy viscosity model indirectly solve Reynolds stress. It is expressed as a function of turbulent viscosity. The key issue of this method is the calculation of turbulent viscosity. Turbulent viscosity is represented via the Boussinesq assumption. This assumption establishes the relationship between Reynolds stress and mean gradients of the velocity:

$$
-\rho \overline{u_{i}^{\prime} u_{j}^{\prime}}=\mu_{t}\left(\frac{\partial u_{i}}{\partial x_{j}}+\frac{\partial u_{j}}{\partial x_{i}}\right)-\frac{2}{3}\left(\rho k+\mu_{t} \frac{\partial u_{i}}{\partial x_{i}}\right) \delta_{i j}
$$

Where, $\mu_{t}$ : turbulent viscosity, $u_{i}$ : mean velocity, $\delta_{i j}:$ "Kronecker delta" symbol $\left(\mathrm{i}=\mathrm{j}, \delta_{i j}=1\right.$; $\left.i \neq j, \delta_{i j}=0\right), k$ : turbulent kinetic energy. 


$$
k=\frac{\overline{u_{i}^{\prime} u_{i}^{\prime}}}{2}=\frac{1}{2}\left(\overline{u^{\prime 2}}+\overline{v^{\prime 2}}+\overline{w^{\prime 2}}\right)
$$

Adding turbulent dissipation rate equation, it establishes the standard $k-\varepsilon$ model.

$$
\varepsilon=\frac{\mu}{\rho} \overline{\left(\frac{\partial u_{i}^{\prime}}{\partial x_{k}}\right)\left(\frac{\partial u_{i}^{\prime}}{\partial x_{k}}\right)}
$$

In standard $k-\varepsilon$ model, the transport equations about incompressible fluid are as follows:

$$
\left\{\begin{array}{l}
\frac{\partial(\rho k)}{\partial t}+\frac{\partial\left(\rho k u_{i}\right)}{\partial x_{i}}=\frac{\partial}{\partial x_{i}}\left[\left(\mu+\frac{\mu_{t}}{\sigma_{k}}\right) \frac{\partial k}{\partial x_{j}}\right]+G_{k}-\rho \varepsilon \\
\frac{\partial(\rho \varepsilon)}{\partial t}+\frac{\partial\left(\rho \varepsilon u_{i}\right)}{\partial x_{i}}=\frac{\partial}{\partial x_{i}}\left[\left(\mu+\frac{\mu_{t}}{\sigma_{\varepsilon}}\right) \frac{\partial \varepsilon}{\partial x_{j}}\right]+\frac{C_{1 \varepsilon}}{k} G_{k}-C_{2 \varepsilon} \rho \frac{\varepsilon^{2}}{k}
\end{array}\right.
$$

Where, $G_{k}$ : generation of turbulence kinetic energy due to the mean velocity gradients. $C_{1 \varepsilon}$ and $C_{2 \varepsilon}$ are constant. $\sigma_{k}$ and $\sigma_{\varepsilon}$ are turbulence Prandtl numbers.

2. LES model

LES directly solves the instantaneous Navier-Strokes equation. Unlike DNS, LES resolves only large eddies directly, while small eddies are modeled. The effect of small eddies is represented by adding an item to LES equations. So, LES can use much coarser grid than DNS. Commercial software Fluent has a LES module for user (Fluent User's Guide).

LES governing equations are obtained by using filtered function that effectively filters out the small eddies. A filter function is defined by:

$$
\bar{\phi}=\int_{D} \phi G\left(x, x^{\prime}\right) d x^{\prime}
$$

Where $D$ is fluid domain, $G\left(x, x^{\prime}\right)$ is the filter function that determines the scale of eddies. $G\left(x, x^{\prime}\right)$ has variable expressions. For finite volume method, the filter function can be defined by:

$$
G\left(x, x^{\prime}\right)=\left\{\begin{array}{l}
1 / V, x^{\prime} \in v \\
0, x^{\prime} \notin v
\end{array}\right.
$$

Where $V$ is the volume of computational cell. So equation (4.24) can be written as followed:

$$
\bar{\phi}=\frac{1}{V} \int_{D} \phi d x^{\prime}
$$

LES equations for incompressible fluid are obtained by using equation (4.26) to filter the Navier-Strokes equations:

$$
\left\{\begin{array}{c}
\frac{\partial \overline{u_{i}}}{\partial x_{i}}=0 \\
\frac{\partial}{\partial}\left(\rho \overline{u_{i}}\right)+\frac{\partial}{\partial}\left(\rho \overline{u_{i}} \bar{u}_{j}\right)=\frac{\partial}{\partial x_{j}}\left(\mu \frac{\partial \delta_{i j}}{\partial x_{j}}\right)-\frac{\partial \bar{p}}{\partial x_{j}}-\frac{\partial \tau_{i j}}{\partial x_{j}}
\end{array}\right.
$$


Where $\tau_{i j}$ is subgrid-scale stress which is defined by equation (4.28). $\delta_{i j}$ is stress tensor.

$$
\tau_{i j}=\rho \overline{u_{i} u_{j}}-\rho \overline{u_{i}} \overline{u_{j}}
$$

The Subgrid-scale stress also complies the Boussinesq hypothesis:

$$
\tau_{i j}-\frac{1}{3} \tau_{k k} \delta_{i j}=-2 \mu_{t} \overline{S_{i j}}
$$

Where $\mu_{t}$ is subgrid-scale turbulent viscosity. $\overline{S_{i j}}$ is the rate-of-strain tensor. In Smagorinsky-Lilly model, $\mu_{t}$ and $\overline{S_{i j}}$ are defined by:

$$
\begin{gathered}
\mu_{t}=\rho L_{s}^{2}|\bar{S}| \\
\overline{S_{i j}}=\frac{1}{2}\left(\frac{\partial \overline{u_{i}}}{\partial x_{i}}+\frac{\partial \overline{u_{i}}}{\partial x_{j}}\right)
\end{gathered}
$$

Where, $|\bar{S}|=\sqrt{2 \overline{S_{i j}} \overline{S_{i j}}}, L_{s}=\min \left(\kappa d, C_{s} V^{\frac{1}{3}}\right)$

$\kappa$ is von karman constant, $d$ is the distance to the closest wall, $C_{s}$ is Smagorinsky constant.

\subsection{Fatigue assessment methods}

Cyclic loading can cause the fatigue damage of SCR. The S-N curve and linear cumulative damage law (Palmrgen-Miner rule) are used to calculate the fatigue life of SCR in offshore industry (DNV-RP-C203, 2010).

The basic $\mathrm{S}-\mathrm{N}$ curve is defined as:

$$
\log N=\log a-m \log \Delta \sigma
$$

Where $N$ is predicted number of cycles to failure for stress range $\Delta \sigma . m$ is negative inverse slope of S-N curve. $\log \bar{a}$ is intercept of long $\mathrm{N}$-axis by S-N curve.

The fatigue strength of welded joints is partly dependent on plate thickness. The S-N curve accounting for the effect of thickness is defined by:

$$
\log N=\log \bar{a}-m \log \left(\Delta \sigma\left(\frac{t}{t_{r e f}}\right)^{k}\right)
$$

Where $t_{r e f}$ is reference thickness. $t$ is thickness through which a crack will mostly grow. $k$ is thickness exponent on fatigue strength.

According to Palmrgen-Miner rule, the fatigue damage is calculated by (ABS, 2003):

$$
D=\sum_{i=1}^{k} \frac{n_{i}}{N_{i}} \leq \eta
$$

Where $D$ is accumulated fatigue damage. $n_{i}$ is the number of stress cycles stress block i. $\eta$ is allowable damage ratio. 
The value of parameters in Equation (4.26) can refer to the standards published by each Classification Society, such as DNV, ABS and CCS.

\subsection{Conclusion}

This section introduces the factors inducing SCR fatigue and mainly introduces the calculation methods of wave force and VIV that are commonly used in offshore industry. Wave force on SCR is obtained by Morsion's equation, which is widely used in calculation of wave loading on slender structure. VIV prediction model can be classified into empirical model and CFD model. Empirical models have been developed for past decades. Commercial software is developed, such as Shear7, VIVA and VIVNA. With the development of computer technology and increased storage, CFD application is growing faster recently. Although the decades of research on VIV and wave induced fatigue, there are uncertainties about the prediction of SCR fatigue life. It is usually to select larger safety coefficient in SCR design.

\section{SCR installation}

SCR installation needs to consider the effects of vessel's motion, wave, current and the interface with other structures. Some factors are discussed above. So this section only introduces the SCR installation methods and equipments. Giving a mechanical model of SCR can be used in installation.

\subsection{The introduction of SCR installation}

SCR installation is a procedure that connects to the pipe laying. Three pipe laying methods are S-lay, J-lay and Reel-lay. These three pipelay ships are shown in figure 5.1. And some SCR installation equipments are shown in figure 5.2, including tensioner, $A \& R$ winch and integrated riser lifting system (Mao et al. , 2010; Duan et al. , 2011).

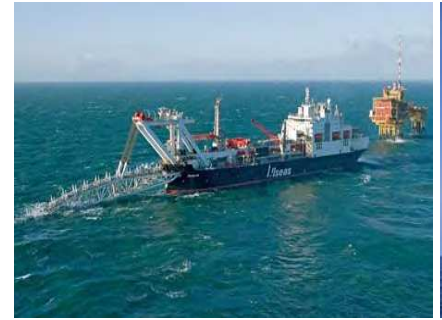

(a) S-lay ship

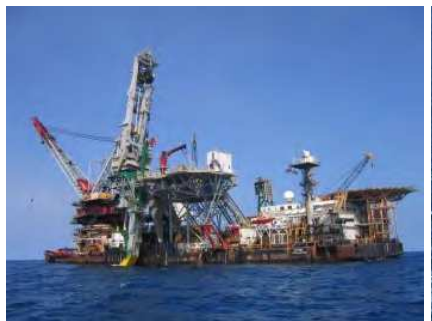

(b) J-lay ship

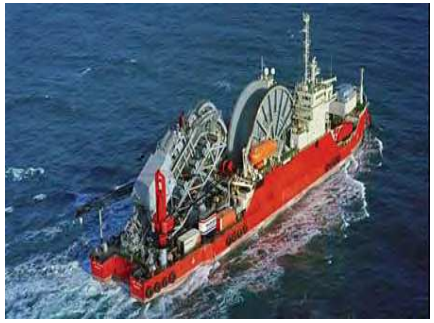

(c) Reel-lay ship

Fig. 5.1 Three pipelay ships

The installation method can be classified into 2 broad categories: $1^{\text {st }}$ installation and $2^{\text {nd }}$ installation. $1^{\text {st }}$ installation is that the pipelay ship starts pipe laying from the platform and lays in the opposite direction to platform, as shown in figure 5.3 (a). $2^{\text {nd }}$ installation has 2 methods: pre-lay and post-lay. Pre-lay method means laying the pipe on the seabed after pipe laying is finished, while the platform is not in position. Then installation vessel lifts the pipe from the seabed and installs the pipe on the platform when the platform is in position. Post-lay method (Smith, 2007) means directly install the pipe on the platform after the pipelay ship finished pipe laying when the platform is in position. Figure 5.3(b) and (c) show the procedure of pre-lay and post-lay. 


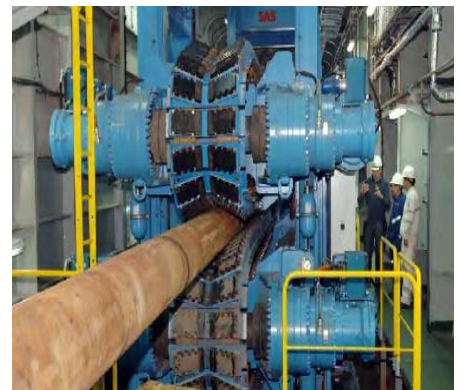

(a) tensioner

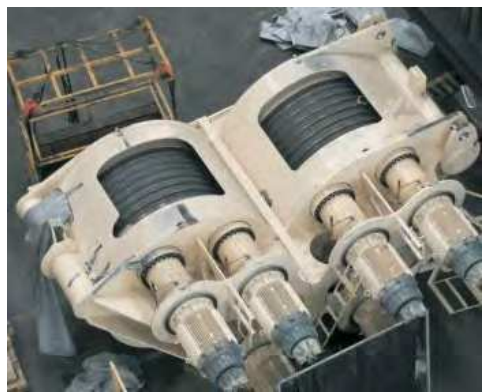

(b) A\&R winch

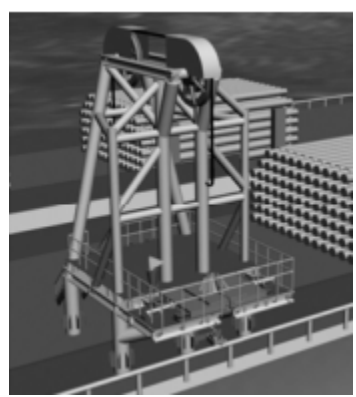

(c) Integrated riser lifting system

Fig. 5.2 SCR installation equipments

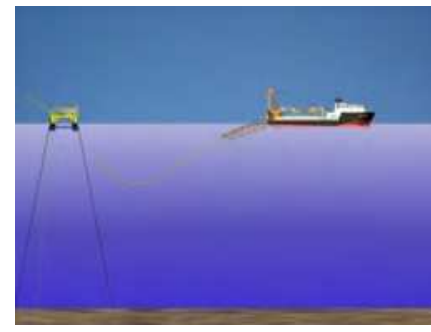

(a) $1^{\text {st }}$ installation

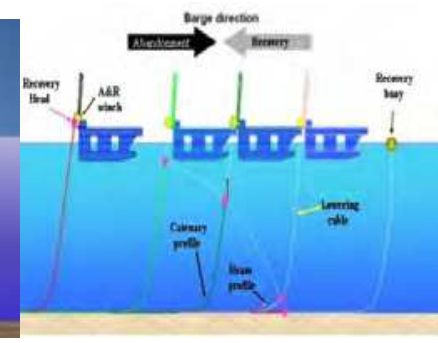

(b) pre-lay method

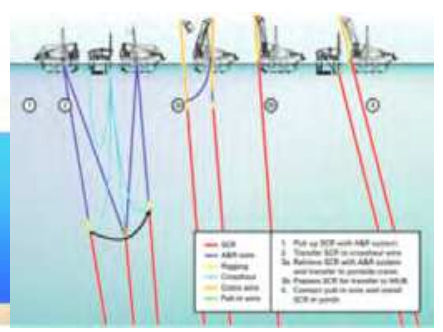

(c) post-lay method

Fig. 5.3 SCR installation methods

\subsection{Mechanical model of SCR}

SCR installation need to consider some factors: installation vessel's movement, environment loadings and the interface with other offshore structures etc. This section only introduces a model of SCR during installation due to the other factors mainly discussed in above sections.

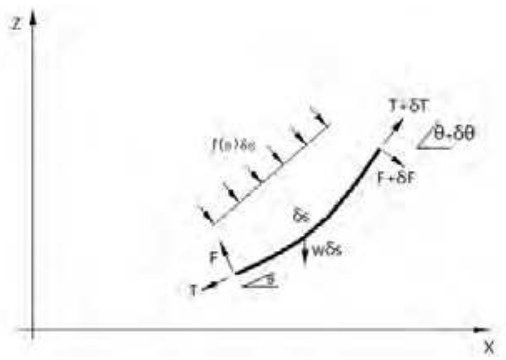

Fig. 5.4 The forces acting on a short segment of SCR

SCR is a large deformation slender structure during installation. It belongs to large deformation and small strain problem. Slender rod and straight beam method can't model catenary riser. This section introduces a Large-Angle deflection beam model (Sparks, 2007). Figure 5.4 shows the forces acting on a short segment of SCR. According to the force balance, the differential equations can be deduced: 


$$
\left\{\begin{array}{l}
E I \frac{d^{3} \theta}{d s^{3}}-T \frac{d \theta}{d s}+w \cos \theta+f(s)=0 \\
\frac{d T}{d s}=w \sin \theta
\end{array}\right.
$$

Where, $f(s)$ is external loading. EI is the constant bending stiffness. $\theta$ is a angle measured from horizontal. $T$ is axial tension.

\subsection{Conclusion}

This section introduces SCR installation methods and equipments. Giving a mechanical model of SCR can be used in SCR installation. The factors affecting SCR installation are discussed in the above sections.

\section{Conclusion}

SCR plays an important role in connecting the floating structure and submarine production facilities. SCR has advantages of low manufacturing cost, resistance of high temperature and high pressure, good adaptability of upper floating body's motion, etc. SCR numerical simulation has made great advances through the decades of research. Some commercial software was developed for SCR design. Despite decades of researches, there are uncertainties on mechanical characteristic of SCR. These are challenges for SCR design and future endeavor is suggested as follows:

1. Pipe-soil interaction mechanism. Pipe-soil interaction models are obtained from experiments. Empirical models have been established. Non-degradation models have been applied in SCR strength analysis and the prediction of fatigue damage. From the field observation, it will develop a trench beneath the SCR under cyclic loading. The development of trench is a difficulty. The formation of trench is affected by complex factors, such as soil plastic deformation, water mixed, soil reconsolidation and soil erosion. Only infering the trench shape will not reach to precise calculation results.

2. Turbulence is recognized as a difficult problem in the world. Turbulent flows exist in our lives: Smoke comes from a chimney, water flows in a river and a strong wind strikes the structure. Turbulent flow is unsteady, irregular, random and chaotic. So, despite decades of intensive research, there are no models that can accurately simulate the turbulent flow. The challenge of VIV of SCR is the calculation of hydrodynamic force under turbulent flow. Empirical models have been used for SCR design. Some commercial software has been developed, such as Shear7, VIVA. Another approach is CFD technology. In theory, hydrodynamic force can be accurately calculated by directly solving Navier-Strokes equations. This method is also called Direct Numerical Simulation (DNS). But DNS requires very refinement grid and micro time step, and the requirement of storage and computation speed is very high. So RANS and LES are widely applied in engineering. The future works should be done to establish more exact model.

3. With the increase of water depth, the coupled effects between hull and riser cannot be neglected. Any uncoupled or simple coupled analysis can't reflect the coupling effect. Now there exists systematic method to conduct the couple analysis. But how to deal with the nonlinear wave load and geometric nonlinearity of the slender structure is still a problem. Although frequency domain analysis can demonstrate the wave response of 
platform to a certain degree and its calculation efficiency is relative high, time domain analysis is the better way to reflect all the nonlinearities. As a result, some software whose main task is the coupled analysis such as the HARP and Cable3D are based on time domain analysis.

\section{Acknowledgement}

This chapter is financially supported by the National 863 Program of China (granted number 2006AA09A105), and funded by the National Natural Science Foundation of China (granted number 50979113). Sincerely thanks go to the colleagues in the COOEC Ltd. and in the Offshore Oil/Gas Research Centre of China University of Petroleum, who are involved in this wide range of researches.

\section{References}

[1] Yong Bai and Qiang Bai. Subsea Pipelines and Risers. Elsevier Science Ltd, 2005.

[2] Rafik Boubenider. Effectiveness of Polyethylene Helical Strakes in Suppressing VIV Responses after Sustaining High Roller Load Deformation during S-Lay Installation. Offshore Technology Conference, 2008.

[3] Samuel Taggart and Michael A.Tognarelli. Offshore Drilling Riser VIV Suppression DevicesWhat's Available to Operators. Offshore Mechanics and Arctic Engineering, 2008.

[4] W.K.Kavanagh, K.R.Farnsworth and P.G.Griffin. Matterhorn Steel Catenary Riser: Critical Issues and Lessons Learned for Reel-Layed SCRs to a TLP. Offshore Technology Conference, 2004.

[5] Abaqus Analysis User's Manual 6.9.

[6] ANSYS ASAS Brochre.

[7] Orcaflex Manual Version 9.3c.

[8] Ali Nakhaee. Study of the fatigue fatigue life of steel catenary risers in interaction with the seabed. Ph.D dissertation of Texas A\&M University, 2010.

[9] M.S.Hodder and B.W.Byrne. 3D experiments investigating the interaction of a model SCR with the seabed. Applied Ocean Research, 2009.

[10] N.R.T.Willis and P.T.J.West. Interaction between Deepwater Catenary Risers and a Soft Seabed: Large Scale Sea Trials. Offshore Technology Conference, 2001.

[11] C.Aubeny and G.Biscontin. Interaction Model for Steel Compliant Riser on Soft Seabed. Offshore Technology Conference, 2008.

[12] Christoper Bridge and Katherine Laver. Steel Catenary Riser Touchdown Point Vertical Interaction Models. Offshore Technology Conference, 2004.

[13] Ali Nakhaee and Jun Zhang. Trenching effects on dynamic behavior of a steel catenary riser. Ocean Engineering, 2009.

[14] E.Fontaine, J.F.Nauroy, P.Foray, A.Roux, H.Gueveneux. Pipe- soil interaction in soft kaolinite: vertical stiffness and damping. In: Proceedings of the 14th International Offshore and Polar Conference, 2004.

[15] Ali Nakhaee and Jun Zhang. Dynamic Interaction Between SCR And Seabed. Offshore Mechanics and Arctic Engineering, 2007.

[16] Paulling, J.R, and Webster, W.C. A Consistent, large-amplitude analysis of the coupled response of a TLP and Tendon system. Proceedings Fifth International Mechanics and Arctic Engineering Symposium,1986. 
[17] Arcandra Tahar and M.H.Kim. Hull/mooring/riser coupled dynamic analysis and sensitivity study of a tanker-based FPSO. Applied Ocean Research. 2003.

[18] Zhang Fan, Yang Jian-min, Li Run-pei, Chen Gang. Coupling effects for cell-truss spar platform: Comparison of frequency- and time-domain analysis with model tests. 2008.

[19] Xiaohong Chen. Studies on dynamic interaction between deep-water floating structures and their mooring/tendon systems. Ph.D dissertation of Texas A\&M University, 2002.

[20] D.L.Garrett. Dynamic analysis of slender rods. Journal of energy resources technology, 1982.

[21] D.L.Garrett. Coupled analysis of floating production systems. Ocean Engineering, 2005.

[22] Arcandra. Hull/Mooring/Riser coupled dynamic analysis of a deepwater floating platform in with polyester lines. Ph.D dissertation of Texas A\&M University, 2001.

[23] Zhihuang Ran. Coupled dynamic analysis of floating structures in waves and currents. Ph.D dissertation of Texas A\&M University, 2000.

[24] HARP manuals user's manual.

[25] Y.M.Low and R.S.Langley. Time and frequency domain coupled analysis of deepwater floating production systems. Applied Ocean Research, 2006.

[26] Morison J R, O'Brien M D, Johnson J W, and Schaaf S A. The force exerted by surface waves on piles. Petrol Trans AIME, 1950.

[27] R.Burrows, R.G.Tickell, D. Hames and G.Najafian. Morison wave force coefficients for application to random seas. Applied Ocean Research, 1997.

[28] C.H.K. Williamson and R. Govardhan. A brief review of recent results in vortex-induced vibrations. Journal of Wind Engineering and Industrial Aerodynamics, 2008.

[29] Pan Zhi-yuan, Cui Wei-cheng and Zhang Xiao-ci. An Overview on VIV of Slender Marine Structures. Journal of Ship Mechanics, 2005.

[30] M.L.Facchinetti, E.de Langre and F.Biolley. Coupling of structure and wake oscillators in vortex-induced vibrations. Journal of fluids and structures, 2003.

[31] Xu Wan-hai, Wu Ying-xiang and Yu Jian-xing. A new wake oscillator model for predicting vortex induced vibration of a circular cylinder. Journal of Hydrodynamics, 2010.

[32] J.Kim Vandiver and Li Li. SHEAR7 V4.4 PROGRAM THEORTICAL MANUAL. 2005.

[33] Z.Y.Pan, W.C.Cui and Q.M.Miao. Numerical simulation of vortex-induced vibration of a circular cylinder at low mass-damping using RANS code. Journal Fluids and Structures, 2007.

[34] Joseph Thomas Klamo. Effects of Damping and Reynolds Number on Vortex-Induced Vibrations. Ph.D dissertation of California Institute of Technology, 2007.

[35] M.Dixon and D.charlesworth. Application of CFD for Vortex-Induced Vibration Analysis of Marine Risers in Projects. Offshore Technology Conference, 2006.

[36] Fluent User's Guide.

[37] DNV-RP-C203. FATIGUE DESIGN OF OFFSHORE STEEL STRUCTURES. 2010.

[38] ABS. Guide for the fatigue assessment of offshore structures, 2003.

[39] Charles P. Sparks. Fundamentals of Marine Riser Mechanics: Basic Principle and Simplified Analyses. Penn Well, 2007.

[40] Christopher E. Smith. SPECIAL REPORT: Independence Hub sees record SCR installation. Oil \& Gas Journal, 2007.

[41] DUAN Meng-lan, WANG Yi, Segen ESTEFEN, HE Ning, LI Li-na and CHEN Bang-min. An Installation System of Deepwater Riser by An S-Lay Vessel. China Ocean Engineering, 2011.

[42] Mao Dongfeng, Duan Menglan, Wang Yi, He Ning, Chen Bangmin and Zhang Yingjie. Model test investigation on an innovative lifting system for deepwater riser installation. PETROLEUM SCIENCE, 2010. 


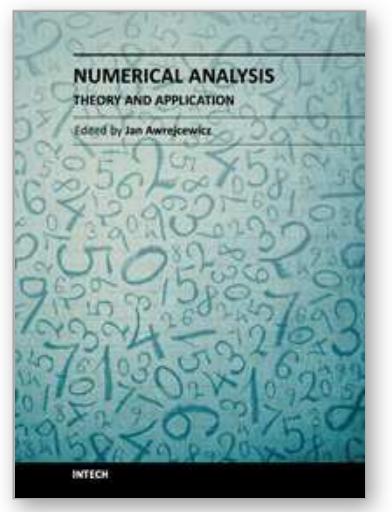

\author{
Numerical Analysis - Theory and Application \\ Edited by Prof. Jan Awrejcewicz
}

ISBN 978-953-307-389-7

Hard cover, 626 pages

Publisher InTech

Published online 09, September, 2011

Published in print edition September, 2011

Numerical Analysis â€“ Theory and Application is an edited book divided into two parts: Part I devoted to Theory, and Part II dealing with Application. The presented book is focused on introducing theoretical approaches of numerical analysis as well as applications of various numerical methods to either study or solving numerous theoretical and engineering problems. Since a large number of pure theoretical research is proposed as well as a large amount of applications oriented numerical simulation results are given, the book can be useful for both theoretical and applied research aimed on numerical simulations. In addition, in many cases the presented approaches can be applied directly either by theoreticians or engineers.

\title{
How to reference
}

In order to correctly reference this scholarly work, feel free to copy and paste the following:

Menglan Duan, Jinghao Chen and Zhigang Li (2011). Mechanics of Deepwater Steel Catenary Riser, Numerical Analysis - Theory and Application, Prof. Jan Awrejcewicz (Ed.), ISBN: 978-953-307-389-7, InTech, Available from: http://www.intechopen.com/books/numerical-analysis-theory-and-application/mechanics-ofdeepwater-steel-catenary-riser

\section{INTECH}

open science | open minds

\section{InTech Europe}

University Campus STeP Ri Slavka Krautzeka 83/A 51000 Rijeka, Croatia Phone: +385 (51) 770447

Fax: +385 (51) 686166 www.intechopen.com

\section{InTech China}

Unit 405, Office Block, Hotel Equatorial Shanghai No.65, Yan An Road (West), Shanghai, 200040, China 中国上海市延安西路65号上海国际贵都大饭店办公楼405单元 Phone: +86-21-62489820

Fax: $+86-21-62489821$ 
(C) 2011 The Author(s). Licensee IntechOpen. This chapter is distributed under the terms of the Creative Commons Attribution-NonCommercialShareAlike-3.0 License, which permits use, distribution and reproduction for non-commercial purposes, provided the original is properly cited and derivative works building on this content are distributed under the same license. 